\title{
Effects of Tumor-Derived Exosome Programmed Death Ligand 1 on Tumor Immunity and Clinical Applications
}

\author{
Bo Shao ${ }^{1,2+}$, Qin Dang ${ }^{1+}$, Zhuang Chen', Chen Chen ${ }^{2,3}$, Quanbo Zhou', Bingbing Qiao4, \\ Jinbo Liu' ${ }^{1}$, Shengyun Hu${ }^{1}$, Guixian Wang ${ }^{1}$, Weitang Yuan ${ }^{1}$ and Zhenqiang Sun ${ }^{1,3 *}$ \\ ${ }^{1}$ Department of Colorectal Surgery, The First Affiliated Hospital of Zhengzhou University, Zhengzhou, China, ${ }^{2}$ Academy \\ of Medical Sciences, Zhengzhou University, Zhengzhou, China, ${ }^{3}$ School of Life Sciences, Zhengzhou University, Zhengzhou, \\ China, ${ }^{4}$ Department of Hepatobiliary and Pancreatic Surgery, The First Affiliated Hospital of Zhengzhou University, \\ Zhengzhou, China
}

\section{OPEN ACCESS}

Edited by:

Christiane Pienna Soares,

São Paulo State University, Brazil

Reviewed by:

Maria Eduarda Battistella,

Federal University of Rio Grande do

Sul, Brazil

Akbar Nawab,

University of Florida, United States

*Correspondence:

Zhenqiang Sun

fccsunzq@zzu.edu.cn

${ }^{t}$ These authors have contributed equally to this work and share first

authorship

Specialty section

This article was submitted to

Epigenomics and Epigenetics, a section of the journal

Frontiers in Cell and Developmental

Biology

Received: 17 August 2021

Accepted: 24 September 2021

Published: 15 October 2021

Citation:

Shao B, Dang Q, Chen Z,

Chen C, Zhou Q, Qiao B, Liu J, Hu S, Wang G, Yuan W and Sun Z (2021) Effects of Tumor-Derived Exosome

Programmed Death Ligand 1 on

Tumor Immunity and Clinical

Applications.

Front. Cell Dev. Biol. 9:760211. doi: 10.3389/fcell.2021.760211
Programmed death ligand 1 (PD-L1) is a typical immune surface protein that binds to programmed cell death 1 (PD-1) on $\mathrm{T}$ cells through its extracellular domain. Subsequently, $T$ cell activity is inhibited, and tumor immune tolerance is enhanced. Anti-PD-1/PD-L1 immune checkpoint therapy blocks the combination of PD-1/PDL1 and rejuvenates depleted T cells, thereby inhibiting tumor growth. Exosomes are biologically active lipid bilayer nanovesicles secreted by various cell types, which mediate signal communication between cells. Studies have shown that PD-L1 can not only be expressed on the surface of tumor cells, immune cells, and other cells in the tumor microenvironment, but also be released from tumor cells and exist in an extracellular form. In particular, exosome PD-L1 plays an unfavorable role in tumor immunosuppression. The immunomodulatory effect of exosome PD-L1 and its potential in fluid diagnosis have attracted our attention. This review aims to summarize the available evidence regarding the biological characteristics of exosome PD-L1 in tumor immunity, with a particular focus on the mechanisms in different cancers and clinical prospects. In addition, we also summarized the current possible and effective detection methods for exosome PD-L1 and proposed that exosome PD-L1 has the potential to become a target for overcoming anti-PD-1/PD-L1 antibody treatment resistance.

Keywords: exosome, PD-L1, PD-1, tumor immunity, biomarker

\section{INTRODUCTION}

Programmed cell death-1 (PD-1), also known as CD279, is expressed in a variety of immune cells, including peripheral activated T cells, B cells, and monocytes (Nishimura et al., 1996; Keir et al., 2008). The two known ligands of PD-1 are Programmed death-ligand 1 PD-L1 (B7-H1) and PDL2 (B7-DC) (Blank et al., 2004). PD-L1 is a typical immune surface protein that binds to PD-1

Abbreviations: PD-L1, programmed death ligand 1; PD-1, programmed cell death 1; ILV, intraluminal vesicles; MVE, multiple vesicle endosomes; ESCRT, endosomal complex required for transportation; TAM, tumor-associated macrophages; sPD-L1, soluble PD-L1; DCs, dendritic cells; Tregs, regulatory T cells; NSCLC, non-small cell lung cancer; HNSCC, Head and neck squamous cell carcinoma; PD-L1 (KD) B16-F10, knockout of PD-L1-expressed B16-F10 cells; APCs, antigen presenting cells; CTL, cytotoxic T lymphocytes; HSCs, hematopoietic stem cells; SPR, surface plasmon resonance; PDAC, Pancreatic ductal adenocarcinoma; ELISA, enzyme-linked immunosorbent assay. 
on $\mathrm{T}$ cells through its extracellular domain (Dong et al., 1999). PD-L1 inhibits the activity of $T$ cells and enhances the immune tolerance of tumor cells, thereby preventing the immune response, which may damage the tumor, and leading to the immune escape of the tumor. PD-L1 was the first PD-1 ligand to be discovered. Numerous studies have shown that PD-L1 is abnormally expressed in many tumors, such as skin, brain, thyroid, esophageal, and colorectal tumors (Lin et al., 2015; Wang et al., 2016; Zhou et al., 2017). Therefore, PD-L1 is considered to be a critical factor involved in tumor immune escape.

Exosomes are biologically active extracellular vesicles approximately 30-120 nm in diameter with a lipid bilayer structure that are secreted by various cells (Théry et al., 2002). Exosomes are intraluminal vesicles (ILVs) formed by inward endosomal membrane budding during the maturation of multiple vesicular endosomes (MVEs; Colombo et al., 2014; Hessvik and Llorente, 2018). MVEs can fuse with lysosomes, leading to the degradation of extracellular vesicles and the recycling of their contents, which promotes cellular metabolism. MVEs also fuse with the cell membrane, causing ILVs to be released extracellularly, and these ILVs that are released from the cell are called exosomes (Colombo et al., 2014). Exosomes are released by various cell types and are stably present in all body fluids (Boukouris and Mathivanan, 2015). It has been widely validated that exosome facilitate communication between cells and the exchange of proteins, nucleic acids, and other substances (Lo Cicero et al., 2015; Cordonnier et al., 2017; Mashouri et al., 2019). Exosomes precisely transfer many biological components to target cells and are an effective way to affect gene expression in distant cells. These biological components are wrapped in a double membrane, which is stable even after being transferred to a remote location (Kourembanas, 2015; van Niel et al., 2018). Exosome biogenesis is a complex process. Lipid- and membrane-associated proteins accumulate in discrete membrane microdomains of the MVE, which then recruits soluble components such as cytoplasmic proteins, RNA, DNA, and cytokines (Nolte-'t Hoen et al., 2012; VillarroyaBeltri et al., 2013; Thakur et al., 2014). This process in turn involves important subunits of the endosomal complex required for transportation (ESCRT). Various subunits of ESCRT are involved in the formation of the entire ILV (Colombo et al., 2013). Recent experimental data indicate that interfering with the RNA of genes associated with ESCRT inactivating these proteins and components affect the secretion efficiency and composition of ILVs (Monypenny et al., 2018). However, some researchers have found that after knocking out the ESCRT complex, exosomes containing the marker CD63 are still present, which means that there may be ESCRT-independent ways to produce exosomes (Stuffers et al., 2009). Exosomes are involved in a wide range of processes, such as metabolic reprogramming (Thakur et al., 2020), macrophage M2 polarization (Ono et al., 2020), tissue repair (Roefs et al., 2020), osteogenic differentiation (Colletti et al., 2020), and hair regeneration (Hu et al., 2020). Emerging evidence suggests that tumor cells attenuate antitumor immunity by expressing biologically active PD-L1 on the surface of their secreted exosomes. In this review, we mainly summarize the mechanism of tumor-derived exosome PD-L1 in the context of tumor immunity and its potential significance in distinct tumor types.

\section{BIOGENESIS AND MOLECULAR CHARACTERISTICS OF EXOSOME PD-L1}

Programmed death ligand 1 is a protein that is expressed on the cell surface. Activation of the PD-1/PD-L1 pathway mainly leads to tumor immune escape and promotes tumor cell growth by affecting $\mathrm{T}$ cell tolerance, $\mathrm{T}$ cell apoptosis, and $\mathrm{T}$ cell failure and enhancing Treg cell functions (Ichikawa and Chen, 2005; Mittal et al., 2014; Zhang et al., 2015). PD-L1 is expressed on the surface of tumor cells (Wang et al., 2016) and promotes tumor immune escape (Daassi et al., 2020). However, the tumor immune escape mediated by PD-L1 on the cell surface is temporary and dependent on IFN- $\gamma$. Moreover, the expression time of PD-L1 on the surface of tumor cells is extremely short. PD-L1 is also expressed on the surface of host immune cells, especially tumorassociated macrophages (TAMs). While PD-L1 disappears from the surface of tumor cells, PD-L1 expression on host immune cells is maintained (Noguchi et al., 2017). Therefore, although PD-L1 on the surface of tumor cells plays a certain role in immune escape, the establishment of an immunosuppressive tumor microenvironment is mainly realized by the expression of PD-L1 on the surface of TAMs.

In addition to being expressed on the cell surface, PD-L1 is also released from tumor cells into the extracellular space to become free PD-L1, including exosome PD-L1 and soluble PD-L1 (sPD-L1; Frigola et al., 2011; Theodoraki et al., 2018). However, before the study of exosome $\mathrm{PD}-\mathrm{L} 1$ received attention, the study of extracellular PD-L1 mainly focused on the effect of sPD-L1 on cancer (Xing et al., 2012; Shi et al., 2013; Nagato et al., 2017). Moreover, the total amount of PD-L1 in circulation did not distinguish between soluble and exosome forms. Recent studies have shown that exosome PD-L1 plays an important role in tumor immunosuppression (Ludwig et al., 2017; Chen et al., 2018; Ricklefs et al., 2018). Compared with sPD-L1, exosome PDL1 is not easily degraded by extracellular proteolytic enzymes and can induce $\mathrm{T}$ cell dysfunction and improve stability (Fan et al., 2019). Therefore, it is important to understand how PDL1 on cells is assembled into exosomes. Monypenny et al. (2018) showed that ALIX, a negative regulator of EGFR, regulates the assembly of certain exosome cargo and controls the balance between exosome PD-L1 and cell surface PD-L1. In ALIXknockdown cells, the proportion of PD-L1 present in the MVEs was larger than that in ILVs, and MVEs were easily observed in the budded state, indicating that ALIX is required for the processing of PD-L1 from the MVE membrane into the ILV (Figures 1A,B). Cordonnier et al. (2020) showed that sPD-L1 may not be a reliable biomarker for melanoma compared to exosome PD-L1. In addition, macrophages and dendritic cells (DCs) release exosomes containing PD-L1 (Hong et al., 2016; Ricklefs et al., 2018; Cordonnier et al., 2020). Interestingly, exosomes in the plasma of patients with chronic lymphocytic leukemia were found to be rich in non-coding Y RNA hY4 


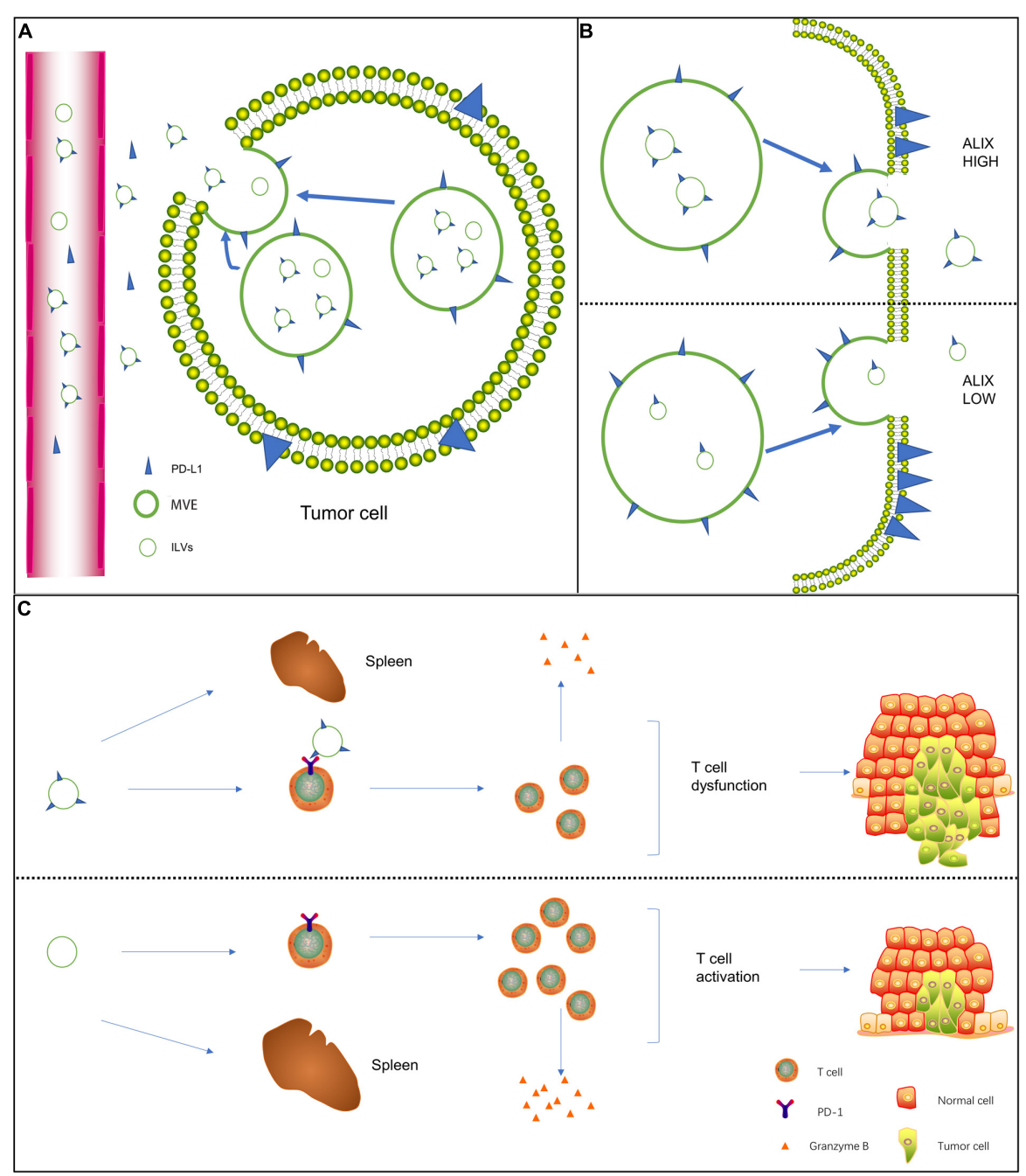

FIGURE 1 | Biogenesis of exosome PD-L1 and its mechanism of action on T cells. (A) In addition to being expressed on the cell surface, free PD-L1 is released from tumor cells to the extracellular space. Free PD-L1 can be divided into exosome PD-L1 and soluble PD-L1. (B) The negative EGFR regulator protein ALIX seems to regulate the assembly of certain exosome cargoes. In ALIX-knockdown cells, PD-L1 expression on the cell surface was strongly increased, but PD-L1 significantly entered exosomes. This decrease indicates that ALIX controls the balance between exosome PD-L1 and cell surface PD-L1. (C) In the presence of exosome PD-L1, the number of activated $T$ cells decreased, the activity of these cells was significantly reduced, the spleen was reduced in size, the level of granzyme B secreted by $T$ cells was significantly reduced, the killing ability of $\mathrm{T}$ cells was inhibited, and tumor growth was significantly promoted.

(Haderk et al., 2017). Additionally, transfer of CLL derived exosomes or hY4 alone to monocytes could lead to key CLLassociated phenotypes, which includes the release of cytokines as well as PD-L1 expression. In sum, the connection between tumor or immune cells and exosome PD-L1 may be highly intricate.

\section{TUMOR-DERIVED EXOSOMES PD-L1 CAN REGULATE IMMUNE CELLS TO PROMOTE TUMOR GROWTH}

\section{Effect of Exosome PD-L1 on T Cells}

Programmed death ligand 1 on the cell surface facilitates tumor immune escape by inducing activated $\mathrm{T}$ cell apoptosis, promoting T cell weakness, enhancing the function of regulatory $\mathrm{T}$ (Treg) cells, inhibiting $\mathrm{T}$ cell proliferation, activating damaged $\mathrm{T}$ cells, and stimulating the production of IL2 (Zhang et al., 2015; Sun et al., 2018). Accumulating evidence has shown that exosome PD-L1 also plays a role in tumor immune escape and promotes tumor development by promoting $\mathrm{T}$ cell apoptosis and inhibiting the production of cytokines (Chen et al., 2018; Guo et al., 2019). Similarly, Kim et al. (2019) showed that exosomes containing PD-L1 could be isolated from the plasma of non-small cell lung cancer (NSCLC) patients. Tumor cell-derived exosome PD-L1 interacts with the $\mathrm{PD}-1$ receptor on CD8 T cells, weakening the function of CD8 $\mathrm{T}$ cells, inducing their apoptosis, and promoting tumor immune escape (Kim et al., 2019). Therefore, membrane proteins on the surface of exosomes perform 
functions in a variety of tumors through direct protein-protein interactions (Table 1).

Almost all previous studies on the interaction between PDL1 and T cells were based on the surface PD-L1-mediated immunosuppression model of tumor cells (Iwai et al., 2002; Mittendorf et al., 2014). Whether exosome PD-L1 binds to PD1 on $\mathrm{T}$ cells and inhibits the activity of CD8 T cells remains unknown. Yang et al. (2018) found that exosome PD-L1 was located on the surface of target cells and bound to PD-1, indicating that exosomes transfer functional PD-L1 to other cells. PD-1 and PD-L1 are part of the exosome cargo and modify the surface of exosomes in the serum of head and neck squamous cell carcinoma (HNSCC) patients (Theodoraki et al., 2019). Poggio et al. (2019) showed that in the presence of exosome PD-L1, $\mathrm{T}$ cells in tumor-draining lymph nodes expressed exhaustion markers, and the spleen was reduced in size (Figure 1C). The activation, proliferation, and killing potential of $\mathrm{T}$ cells is significantly enhanced by the removal of exosomes at the genetic level or by the deletion of PD-L1. When exogenous exosome PD-L1 was reintroduced, the effect was reversed (Poggio et al., 2019). Chen et al. (2018) established a mouse melanoma model with knockout of PD-L1 expression in B16-f10 cells [PD-L1 (KD) B16-F10 cells]. After the injection of exosomes derived from parental B16-F10 cells, the growth of tumors derived from PDL1 (KD) B16-F10 cells was promoted, and the number of CD8 $\mathrm{T}$ lymphocytes invading the tumor was downregulated (Chen et al., 2018). The growth of tumor cells stimulated with exosomes containing PD-L1 was considerably increased compared with that of the control group in a constructed mouse breast cancer model (Yang et al., 2018).

\section{Exosome PD-L1 Can Promote Tumor Immune Escape by Inducing Macrophage M2 Polarization}

Macrophages are derived from monocytes, which in turn are derived from precursor cells in the bone marrow. Macrophages are usually used to maintain the homeostasis of the internal environment and resist the invasion of pathogens (Davies et al., 2013). Macrophages in different environments will produce corresponding polarization, such as common M1 macrophages and M2 macrophages (Martinez and Gordon, 2014). M1 macrophages can promote inflammation and release proinflammatory related factors, while M2 macrophages can resist inflammation, play important roles in immunity, tissue homeostasis, metabolism, and endocrine signal transduction, and can promote tumor metastasis and proliferation (Funes et al., 2018).

Previous studies have found that tumor-derived exosomes can induce M2 polarization of macrophages. For example, studies by Gabrusiewicz et al. (2018) have shown that exosomes derived from glioblastoma stem cells can pass through the monocyte cytoplasm and cause muscle activity. The recombination of protein skeleton transformed monocytes into immunosuppressive M2 type, and the expression of PDL1 in macrophages increased. Haderk et al. (2017) found that when exosomes from chronic lymphocytic leukemia transfer to monocytes, they will cause inflammation, lead to cancer, and increase the expression of PD-L1, and make tumor immune escape. According to the above research, the focus of previous researchers is the change of PD-L1 in M2 macrophages and the impact on tumors. However, there is little research on whether PD-L1 in tumor-derived exosomes influences macrophage polarization. Tumor cells increase the release of glutamate through the cystine/glutamate transporter cystine-glutamate exchange $(\mathrm{xCT})$ to balance the oxidation homeostasis in tumor cells and promote tumor progression (Okazaki et al., 2017). The latest study by Liu et al. (2021) found that inhibiting xCT in melanoma can cause the transcription factor IRF4/EGR1 to upregulate the expression of PD-L1, which leads to melanoma cells secreting many exosomes carrying PD-L1, which in turn induces M2 macrophages polarized and reduced the efficacy of anti-PD-1/PD-L1 in the treatment of melanoma. And it was further discovered that sulfasalazine (SAS) induced macrophage M2 polarization through exosome PD-L1, which weakened the anti-PD-1/PDL1 curative effect, and finally led to anti-PD-1/PD-L1 treatment resistance (Liu et al., 2021).

In fact, it is not difficult to see that the expression of PD-L1 is upregulated in different tumor cells. The important mechanism of tumor immune escape is the combination

TABLE 1 | Tumor-derived exosome PD-L1 in cancers.

\begin{tabular}{|c|c|c|c|c|}
\hline Tumor types & Target cell & Mechanisms & $\begin{array}{c}\text { Tumor } \\
\text { progression }\end{array}$ & References \\
\hline NSCLC & T cell & $\begin{array}{l}\text { Exosome PD-L1 inhibits cytokines (IL-2 and IFN- } \gamma \text { ) and induces apoptosis of CD8 }{ }^{+} \\
\text {T-cells }\end{array}$ & $\uparrow$ & Kim et al., 2019 \\
\hline Melanoma & T cell & $\begin{array}{l}\text { Exosome PD-L1 pass through } T \text { lymphocytes of secondary lymphoid organs and } \\
\text { play a role through the immunosuppressive pathway of PD-1/PD-L1 }\end{array}$ & $\uparrow$ & $\begin{array}{l}\text { Cordonnier } \\
\text { et al., } 2020\end{array}$ \\
\hline Gastric cancer & T cell & $\begin{array}{l}\text { Reduces the expression of CD69 and PD-1 on the surface of T cells, resulting in T } \\
\text { cell dysfunction }\end{array}$ & $\uparrow$ & Fan et al., 2019 \\
\hline Breast cancer & T cell & $\begin{array}{l}\text { Blocks phosphorylation of src family proteins, LAT and PLC } \gamma \text { in CD8 T cells, and } \\
\text { promotes CD8 T cell dysfunction }\end{array}$ & $\uparrow$ & $\begin{array}{l}\text { Chatterjee } \\
\text { et al., } 2020\end{array}$ \\
\hline HNSCC & T cell & Exosome PD-L1 inhibits and interferes $\mathrm{CD}^{+}$effector $\mathrm{T}$ cell activation & $\uparrow$ & $\begin{array}{l}\text { Theodoraki } \\
\text { et al., } 2018\end{array}$ \\
\hline
\end{tabular}

$\uparrow$, tumor promotion; NSCLC, non-small cell lung cancer; HNSCC, head and neck squamous cell carcinoma. 
of PD-L1 with PD-1 of T cells, which causes an immune checkpoint response. With the increasing number of studies on exosome PD-L1 recently, its role in research has become more and more important. Related studies have found that the exosome PD-L1 and cell surface PD-L1 have the same membrane topology by using enzyme-linked immunosorbent assay (Chen et al., 2018). Exosomes containing PD-L1 secreted by tumors can effectively transfer exosomes PD-L1 to macrophages and weaken anti-tumor immunity in tumor microenvironment (Yang et al., 2018). However, the mechanism of how exosome PD-L1 induces immunosuppression has not yet been fully elucidated. Liu et al. (2021) confirmed that by increasing the expression of melanoma exosomes PD-L1, the M2 polarization of macrophages can be induced, which ultimately leads to resistance to anti-PD-1/PD-L1 treatment. In addition, this method is consistent with the results of tumor immune escape and anti-PD-L1 treatment caused by directly upregulating PDL1 in macrophages (Zhang et al., 2017; Wen et al., 2018). All these indicate that tumor-derived exosomes PD-L1 can promote tumor immune escape by inducing the polarization of macrophages M2.

\section{IMMUNOSUPPRESSIVE EFFECTS OF TUMOR-DERIVED EXOSOME PD-L1 ON DISTINCT CANCERS}

\section{Non-small Cell Lung Cancer}

Lung cancer is the leading cause of cancer-related death worldwide (Siegel et al., 2012). Various targeted therapies and immunotherapies for NSCLC have been gradually and effectively applied (He et al., 2015; Grigg and Rizvi, 2016). Among them, $\mathrm{PD}-1 / \mathrm{PD}-\mathrm{L} 1$ inhibitors are representative and have improved the clinical efficacy of NSCLC treatment to a certain extent (Herbst et al., 2016; Brahmer et al., 2017; Mok et al., 2019). Measurement of the expression level of exosome PD-L1 plays a fundamental role in the diagnosis and prognosis of NSCLC. However, there is still no expected response of NSCLC patients with positive immunohistochemical staining for PD-L1 to immunotherapy, and the reason remains elusive. Li et al. (2019) showed that exosome PD-L1 levels were significantly higher in NSCLC patients (especially in advanced-stage individuals) than in healthy controls. The level of exosome PD-L1 was obviously related to tumor size, positive lymph node status, distant metastasis and TNM stage (Li et al., 2019). Kim et al. (2019) found that exosomes containing PD-L1 could be isolated from the plasma of patients with NSCLC. Exosome PD-L1 plays an important role in tumor immune escape by inhibiting cytokines and inducing CD8 T cell apoptosis. Liu et al. measured exosome PD-L1 expression in NSCLC patients using a compact surface plasmon resonance (SPR) biosensor and found that exosome PD-L1 expression was significantly higher than that in healthy controls (Liu et al., 2018). Exosome PD-L1 from NSCLC cells has also been shown to mediate immune escape by inhibiting cytokines (IL-2 and IFN$\gamma$ ) and inducing CD8 T cell apoptosis (Kim et al., 2019). In short, exosome PD-L1 may be a novel biomarker and a promising target for lung cancer.

\section{Melanoma}

Melanoma is a typical immunosuppressive malignant tumor with a high possibility of distant metastasis (Aubuchon et al., 2017). At present, immune checkpoint inhibitor therapy targeting PD-L1 has made remarkable achievements in the treatment of melanoma (Chen and Han, 2015; Topalian et al., 2016). However, the currently approved response rate of patients with advanced melanoma to monoclonal antibodies is still not satisfactory (Ribas et al., 2016; Zaretsky et al., 2016). Therefore, the identification of a typical biomarker is critical for the diagnosis and treatment of melanoma. The original intention was to focus on PD-L1 expression in tumors and blood samples; however, these test results were not necessarily reliable due to the inhibition of PD-L1 in tumors and the instability of PD-L1 in blood. Recently, exosome PD-L1 has been identified as a potential biomarker of melanoma. Cordonnier et al. (2020) further confirmed that circulating exosome PD-L1 in melanoma patients plays a role through $\mathrm{T}$ lymphocytes in secondary lymphoid organs and through the immunosuppressive PD-1/PD-L1 pathway. Moreover, a large increase in exosome PD-L1 is related to tumor progression (Cordonnier et al., 2020). Chen et al. (2018) demonstrated the presence of melanoma-associated exosome PD-L1 and its immunosuppressive effects and suggested that the exosome $\mathrm{PD}-\mathrm{L} 1$ level is an indicator to distinguish clinical responders from non-responders.

\section{Gastric Cancer}

Gastric cancer is the fourth most common cancer in the world (Xiang et al., 2016). The efficacy of anti-PD-1 therapy in metastatic gastric cancer seems to be quite promising (Muro et al., 2016). A recent study have shown that exosome PD-L1 is required to predict the prognosis of gastric cancer patients. Fan et al. (2019) showed that there was a significant correlation between the level of exosome PD-L1 and the stage of gastric cancer, and the survival rate was worse in the group with higher exosome PD-L1 expression. The OS of patients with high exosome PD-L1 expression was significantly lower than that of patients in the low expression group with both stages I and II AJCC, demonstrating the predictive value of exosome PD-L1 for the OS of patients with early gastric cancer.

\section{Breast Cancer}

HER2 expression is elevated in $25 \%$ of breast cancer patients and is often accompanied by a poor prognosis (Slamon et al., 1989). At present, breast cancer treatments targeting HER2 have achieved some efficacy in clinical practice. However, not all breast cancer patients who overexpress HER2 respond to therapy, and many patients still develop treatment resistance (Nahta and Esteva, 2007). Notably, enhanced drug resistance is likely to cause the immune escape of cancer cells (Bruttel and Wischhusen, 2014). Chatterjee et al. (2020) reported that breast cancer cells secrete exosomes carrying PD-L1 and are highly immunosuppressive. Additionally, exosome PD-L1 is stimulated by TGF- $\beta$, which blocks the phosphorylation of src family proteins and promotes CD8 T cell dysfunction. Therefore, 
exosome PD-L1 has considerable potential for the diagnosis and treatment of breast cancer patients (Martinez et al., 2017).

\section{Pancreatic Cancer}

Pancreatic cancer is one of the cancers with the highest mortality rate, and the detection of exosome PD-L1 in the blood is a good prognostic indicator of pancreatic cancer. Pancreatic ductal adenocarcinoma (PDAC) is the most common histological subtype of malignant pancreatic cancer (Rahbari et al., 2016), accounting for $90 \%$ of all cases. Because this type of malignant tumor is highly invasive and infiltrative, most diagnoses are made at the advanced tumor stage. The presence of a tumor immune escape mechanism leads to the rapid development of pancreatic cancer. To date, patients with pancreatic cancer have hardly responded to monotherapy with checkpoint inhibitors (Foley et al., 2016). PD-L1 is highly expressed in pancreatic cancer and is associated with poor prognosis (Geng et al., 2008; Chen et al., 2009; Wang et al., 2010). Therefore, the expression of tumor-derived exosome PD-L1 will greatly improve the diagnostic status of pancreatic cancer and immunotherapy. Lux et al. (2019) examined the expression of PD-L1 in blood samples and showed that the survival time of PD-L1-positive patients was significantly lower than that of PD-L1-negative patients. Therefore, the expression of PD-L1 in exosomes has profound significance for the prognosis of pancreatic cancer. However, since exosome PD-L1 expression in CP patients is higher than that in PDAC patients, exosome PD-L1 may not be suitable as a diagnostic indicator for pancreatic cancer (Lux et al., 2019).

\section{Head and Neck Squamous Cell Carcinoma}

Head and neck squamous cell carcinoma is a common and lethal disease with the highest diagnostic rate in the world (Ferlay et al., 2013; Mourad et al., 2017). The tumor microenvironment of HNSCC has strong immunosuppressive properties, and HNSCC is a highly immunosuppressive, malignant tumor (Ferris et al., 2006; Bergmann et al., 2007; Whiteside, 2018). The PD-1/PDL1 immunosuppressive pathway has received great attention. A series of experimental studies showed that patients with excessive plasma exosome PD-L1 had higher disease activity than patients with lower exosome PD-L1 levels. Higher plasma levels of exosome PD-L1 were associated with stronger inhibition of CD8 effector T cell activation, and anti-PD-1 Abs significantly reduced the dose-dependent effect of exosome PD-L1 on T cell activity and sex-associated inhibition (Theodoraki et al., 2018). Therefore, in HNSCC, exosome PD-L1 binds to the $\mathrm{PD}-1$ receptor on the surface of activated $\mathrm{T}$ cells to maintain their biological activity and effectively transmit signals, thereby affecting the function of immune cells and leading to immune escape. The level of tumor-derived exosomes can be used as an indicator to reflect the efficacy of patient treatment (Theodoraki et al., 2019). Theodoraki et al. (2018) isolated exosomes carrying PD-L1 from the plasma of HNSCC patients and inhibiting $\mathrm{T}$ cell function, demonstrating that circulating exosome PDL1 may be a useful indicator of disease and immune activity in HNSCC patients.

\section{Lymphoma}

Lymphoma is a primary malignant tumor of lymph nodes or lymph tissues, and its occurrence may be related to gene mutations (Zahn et al., 2020), virus and other pathogen infections (Huang et al., 2021). Studies highlight the roles of clonally diverse $\mathrm{CD} 4 \mathrm{~T}$ cells and innate effectors in the efficacy of PD-1 blockade in classical Hodgkin lymphomas (Cader et al., 2020). Additionally, study show that diffuse large B-cell lymphomas possess a self-organized infrastructure comprising side population (SP) and non-SP cells, where transitions between clonogenic states are modulated by exosome-mediated WNT signaling (Koch et al., 2014). Li et al. evaluated the prognostic value of pretreatment circulating sPD-L1 and exoPD-L1 in extranodal NK/T cell lymphoma patients (Li et al., 2020). Their study revealed that circulating exoPD-L1 and sPD-L1 levels were significantly elevated in extranodal NK/T cell lymphoma and might be promising biomarkers for evaluating the survival outcomes of extranodal NK/T cell lymphoma patients.

\section{FUTURE CLINICAL APPLICATIONS OF EXOSOME PD-L1}

\section{Development of Exosome PD-L1 Detection Methods}

Although the application of immunotherapy has shown considerable value in the diagnosis, treatment, and prediction of various cancers, the response rates of patients with positive PDL1 pathology to immunotherapy is only $10-30 \%$ (Fehrenbacher et al., 2016; Eggermont et al., 2018). The level of exosome PD-L1 can reflect the occurrence of tumors in certain cancer types and has a strong correlation with the response to immunotherapy (Chen et al., 2018). Therefore, the detection of exosome PDL1 levels can be used as a supplement to existing immune checkpoint measurements to increase the accuracy of diagnosis. At present, the accepted quantitative detection method for exosome PD-L1 is enzyme-linked immunosorbent assay (ELISA; Welton et al., 2010; Nawaz et al., 2014; Chen et al., 2018). However, this method has certain limitations. When exosome PD-L1 expression is too low $(<200 \mathrm{pg} / \mathrm{ml})$, it is impossible to distinguish between patients and healthy people (Ramirez et al., 2018). Furthermore, Huang et al. (2020) proposed a detection method called HOLMES-ExoPD-L1 that replaces ELISA to quantitatively detect exosome PD-L1. By applying an aptamer with a higher recognition efficiency than the PD-L1 antibody, the detection sensitivity is significantly improved. The uniformity of thermophoresis is used to promote faster binding of the aptamer to exosome PD-L1 (Huang et al., 2020). Pang et al. (2020) proposed an in vitro assay to detect plasma exosome PD-L1, which is undetectable by ELISA. The principle involves the use of nanoparticles to enrich exosomes by binding the $\mathrm{TiO}_{2}$ shell and the hydrophilic phosphate head of exosome phospholipids. This method efficiently captures up to $96.5 \%$ of exosomes, which are then quantified by labeling exosome PD-L1 with a specifically labeled anti-PD-L1 antibody (Pang et al., 2020). Liu et al. (2018) developed a compact surface plasmon resonance (SPR) biosensor with the same principle as traditional SPR, which is a highly 
sensitive, real-time, label-free optical detection method that does not require nanomaterials and effectively reduces the detection cost. Researchers analyzed NSCLC serum samples with this method and found that the expression of exosome PD-L1 in patients with NSCLC was increased. Surprisingly, this method has a higher detection sensitivity than the traditional ELISA detection method. With the same sample size, the researchers used this method to detect exosome PD-L1 levels that ELISA could not detect (Liu et al., 2018; Table 2).

\section{Early Diagnosis and Prognosis of Cancer}

Surgery is still the preferred method for radical treatment of tumors, but quite a lot of cancer patients are usually diagnosed at the advanced stage, thus missing the best opportunity for treatment. For example, most patients with gastric cancer are usually diagnosed at the advanced stage, and the 5-year survival rate is less than 20\% (Price et al., 2012). 75\% of lung cancer patients are already in the advanced stage when they are discovered (Steinman and Banchereau, 2007). Pancreatic ductal adenocarcinoma (PDAC) is highly aggressive and invasive, most of the diagnoses are performed in the advanced tumor stage (Rahbari et al., 2016). Therefore, exploring reliable indicators for early cancer diagnosis and prognostic factors has far-reaching significance for cancer diagnosis and treatment. Many reports show that PD-L1 is abnormally highly expressed in a variety of tumors (skin, brain, thyroid, esophagus, colorectal, etc.) (Iwai et al., 2002; Taube et al., 2014; Patel and Kurzrock, 2015). However, due to the inhibition of PD-L1 in tumors and the instability of PD-L1 in blood samples, some studies have shown that there is no difference in the concentration of sPD-L1 between NSCLC patients and healthy blood donors (Li et al., 2019). Therefore, simply detecting PD-L1 in tumors or blood is very unreliable for the early diagnosis of tumors. We know that exosomes have been widely regarded as a new type of crosstalk circuit between tumor cells and the tumor microenvironment (Li et al., 2015; Melo et al., 2015; Tang and
Wong, 2015). Some studies have clarified that exosomes even represent the mechanism by which immunosuppressive agents in TME participate in the tumor progression cycle (Whiteside, 2016; Ludwig et al., 2017). Many current studies have shown that the detection of the expression level of exosomes PDL1 is of great significance for the early diagnosis of tumors (Chen et al., 2018; Li et al., 2019). Li et al. (2019) showed that the level of exosome PD-L1 in NSCLC patients (especially advanced patients) was significantly higher than that in healthy controls. The level of exosome PD-L1 was significantly correlated with tumor size, lymph node positive status, distant metastasis and TNM stage (Li et al., 2019). However, the level of sPDL1 is not related to clinicopathological features other than tumor size. Liu et al. (2018) measured the expression level of exosome PD-L1 in NSCLC patients by using a compact surface plasmon resonance (SPR) biosensor and found that the expression of exosome PD-L1 was significantly higher than that in healthy controls. The above studies show that exosome PDL1 may become a promising biomarker for the diagnosis of lung cancer. In the plasma of melanoma patients, the level of exosome PD-L1 was significantly higher than that of sPD-L1, and exosome PD-L1 was detected in all patients. Although the level of exosome PD-L1 has no relationship to clinicopathological features, the change after treatment $(\triangle$ ExoPD-L1) is related to tumor response to treatment, and it is verified that the increase of exosome PD-L1 is related to tumor progression (Cordonnier et al., 2020). Fan et al. (2019) showed that there was a significant correlation between the level of exosome PDL1 and the stage of gastric cancer, and the survival rate was worse in the group with higher exosome PD-L1 expression. The OS of patients with high exosome PD-L1 expression was significantly lower than that of patients in the low expression group with both stages I and II AJCC, demonstrating the predictive value of exosome PD-L1 for the OS of patients with early gastric cancer (Fan et al., 2019). The above-mentioned studies show that the exosome PD-L1 is more reliable than tumor and serum

TABLE 2 | Exosome PD-L1 detection method.

\begin{tabular}{|c|c|c|c|}
\hline Method & Mechanism & Advantage & Disadvantages \\
\hline $\begin{array}{l}\text { Enzyme-linked immunosorbent assay } \\
\text { (ELISA) }\end{array}$ & $\begin{array}{l}\text { The PD-L1 antigen and antibody are } \\
\text { adsorbed on the surface of the solid } \\
\text { phase carrier, allowing the antigen and } \\
\text { antibody to react on the surface }\end{array}$ & Strong specificity, Fast Low cost & $\begin{array}{l}\text { Low sensitivity, When the expression of } \\
\text { PD-L1 in exosomes is too low } \\
\text { ( }<200 \mathrm{pg} / \mathrm{ml} \text { ), it is impossible to } \\
\text { distinguish patients from healthy people }\end{array}$ \\
\hline HOLMES-ExoPD-L1 & $\begin{array}{l}\text { Due to the different depletion rates, the } \\
\text { extracellular aptamers can observe } \\
\text { strong fluorescence }\end{array}$ & $\begin{array}{l}\text { Compared with PD-L1 antibody, the } \\
\text { use of aptamers provides higher } \\
\text { recognition efficiency, which can } \\
\text { significantly improve the detection } \\
\text { sensitivity, The operation is simple }\end{array}$ & $\begin{array}{l}\text { The biological stability of aptamers is } \\
\text { poor compared to antibodies, and the } \\
\text { short half-life in vivo limits the } \\
\text { development of aptamers in clinical } \\
\text { applications }\end{array}$ \\
\hline $\begin{array}{l}\text { Based on } \mathrm{Fe}_{3} \mathrm{O}_{4} @ \mathrm{TiO}_{2} \text { isolation and } \\
\text { SERS immunoassay }\end{array}$ & $\begin{array}{l}\mathrm{Fe}_{3} \mathrm{O}_{4} @ \mathrm{TiO}_{2} \text { nanoparticles are used to } \\
\text { enrich exosomes by combining the }\end{array}$ & $\begin{array}{l}\text { The speed is faster, Exosome PD-L1 } \\
\text { can be captured and analyzed directly }\end{array}$ & $\begin{array}{l}\text { With the use of nanomaterials, the cost } \\
\text { may be higher }\end{array}$ \\
\hline
\end{tabular}

SERS immunoassay

Compact surface plasmon resonance (SPR) biosensor enrich exosomes by combining the

$\mathrm{TiO}_{2}$ shell with the hydrophilic

phosphate head of exosome

phospholipids, followed by the addition

of Au @ Ag @ MBA SERS tag modified

with anti-PD-L1 antibody to mark the

outside exosome PD-L1 for

quantification

The same as the traditional SPR

sensing mechanism can be captured and analyzed directly from the serum
Low sensitivity, When the expression of 1 in exosomes is too low distinguish patients from healthy people

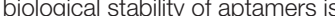
short half-life in vivo limits the

With the use of nanomaterials, the cost ay be higher
High-sensitivity, label-free, real-time optical detection method
Need to use its special equipment, there is a certain learning cost 
PD-L1, and it is of great significance for the early diagnosis and prognosis of cancer.

\section{Exosome PD-L1 as a Biomarker for Clinical Anti-PD-1/PD-L1 Therapy}

Programmed death ligand 1 is rarely expressed on the cell surface of normal human tissues and is abundantly expressed on the surface of cancer cells (Dong et al., 2002). Additionally, IFN$\gamma$ upregulates PD-L1 on the surface of normal tissue cells and cancer cells. The use of an anti-human PD-L1 antibody prevents the effect of tumor cell PD-L1 on activated effector T cells and blocks the interaction of PD-L1 with $\mathrm{T}$ cells. This finding shows that the use of an anti-PD-L1 antibody inhibits the progressive growth of mouse tumors (Dong et al., 2002). PD-L1 inhibits the anti-tumor function of $\mathrm{T}$ cells by activating $\mathrm{PD}-1$. The PD-L1 signaling pathway causes immune escape of tumor cells in the tumor microenvironment (TME; Mittal et al., 2014). Subsequent studies confirmed the accuracy of this concept (Iwai et al., 2002; Strome et al., 2003). These studies showed that tumors evade immune attack through the PD-1/PD-L1 pathway and provide an anti-PD-1/PD-L1 approach for cancer therapy (Figure 2).

Additionally, the FDA has approved two PD-1 antibodies for the treatment of human cancer. Multiple clinical studies have shown that anti-PD-1/PD-L1 therapy has exceedingly significant clinical importance for improving the survival rates of patients with advanced and metastatic tumors. Moreover, in a variety of cancers, especially solid tumors, anti-PD-1/PD-L1 therapy has a fairly long-lasting effect. Notably, the removal of exosome PDL1 inhibits tumor growth, even in models that are resistant to anti-PD-L1 antibodies (Poggio et al., 2019). In some studies of metastatic melanoma, anti-PD-1/PD-L1 therapy showed profound application prospects (Ribas et al., 2016; Topalian et al., 2016). At present, immunohistochemical (IHC) staining of PDL1 is routinely tested in clinical practice to predict the effect of anti-PD-1/PD-L1 immunotherapy (Martinez and Gordon, 2014). Compared with the PD-L1 negative/weak expression group, the remission rate of the $\mathrm{PD}-\mathrm{L} 1 \mathrm{high}$ expression group increased from 8 to 30\% (Funes et al., 2018). But even so, a considerable number of NSCLC patients with positive PD-L1 IHC staining have unsatisfactory immunotherapy effects (Gabrusiewicz et al., 2018). Among them, PD-1/PD-L1 inhibitors are represented, which have improved the clinical efficacy of NSCLC and other tumors to a certain extent (Okazaki et al., 2017; Chen et al., 2018; Liu et al., 2021). However, many tumor patients with positive immunohistochemical staining for PD-L1 still have no expected response after receiving immunotherapy.

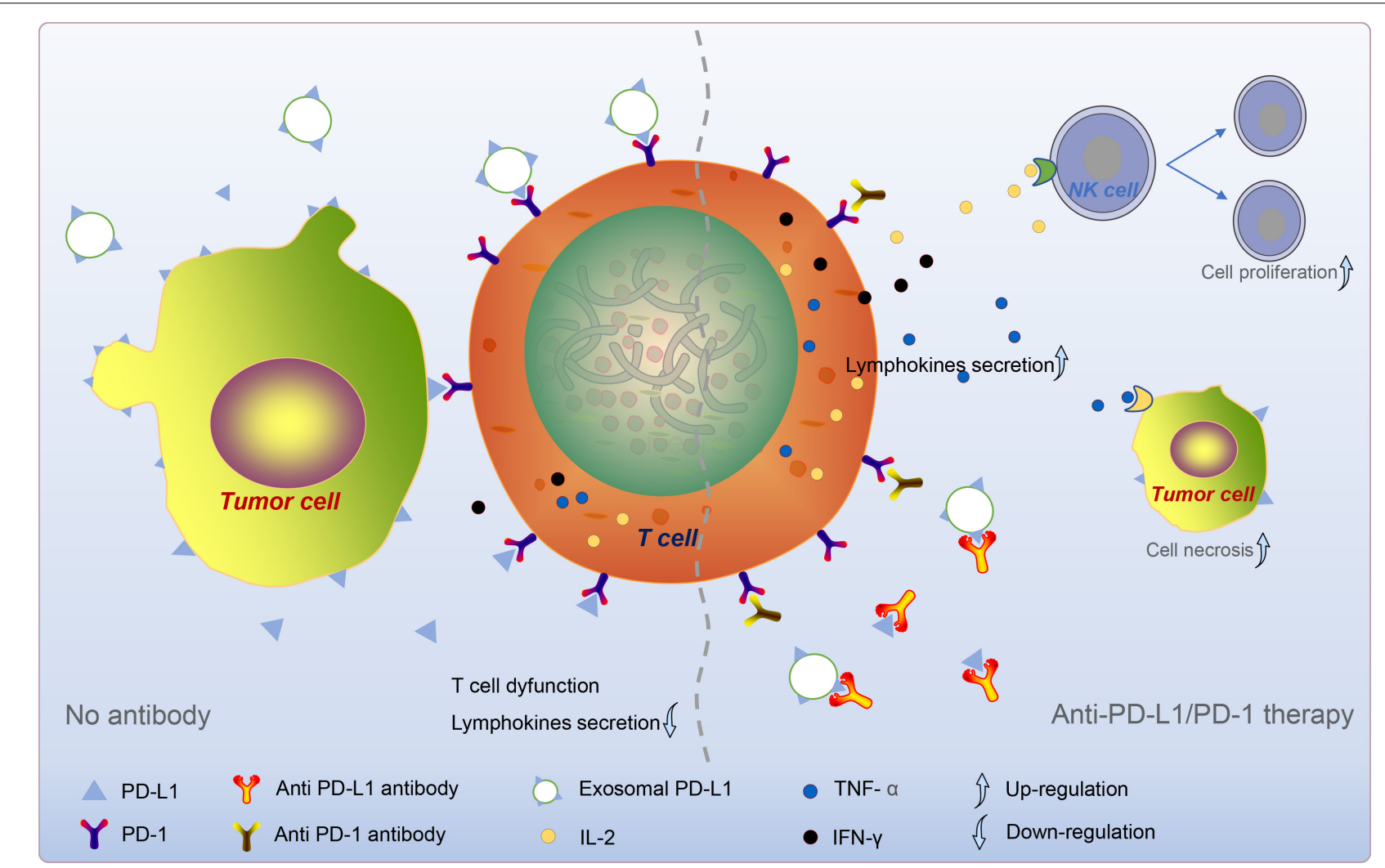

FIGURE 2 | Tumor cell-derived exosome PD-L1-mediated immune escape. Exosome PD-L1, which synergizes with sPD-L1 and PD-L1 on the surface of tumor cells, binds to PD-1 on T cells and reduces T cell activity and lymphokine secretion. In anti-PD-L1/PD-1 therapy, the antibody specifically binds to PD-L1/PD-1, prevents the coupling of circulating PD-L1 to PD-1, increases the secretion of cytokines. Subsequently, these cytokines, such as IL-2, TNF- $\alpha$, and IFN- $\gamma$, could give rise to the division and proliferation of NK cells and the death of tumor cells. The dotted line divides the picture into left and right sides. The left part mainly describes the "No antibodies" situation, and the right part mainly talks about the "anti-pd-1/PD-L1 therapy" situation. 
In gastric cancer patients, not all $\mathrm{PD}-\mathrm{L} 1$ positive patients respond to anti-PD-1, and even PD-L1-negative patients respond (Wen et al., 2018). The reason for this disappointing result is not yet clear, but it is likely that the integrated mechanism of the PD-L1 pathway in TME is not fully understood. Therefore, we need to have a deeper understanding of the immunosuppressive pathway of PD-1/PD-L1 to better improve the treatment efficacy in patients. Recent studies have shown that circulating exosome PD-L1 promotes activated $\mathrm{T}$ cell apoptosis and inhibits the production of cytokines (Chen et al., 2018). Antibodies against PD-L1 or PD-1 block the inhibitory effect of exosome PD-L1 on T cells. Exosome PD-L1 may reflect the dynamic interaction between tumors and immune cells (Chen et al., 2018). In treated patients, the recovery of $\mathrm{T}$ cell activity was negatively correlated with an increase in exosome PD-L1. Exosome PDL1 reflected whether anti-PD-1 therapy successfully triggered anti-tumor immunity. Currently, circulating exosome PD-L1 has been used as a predictive biomarker of the clinical response to anti-PD-1 therapy. However, given that the dynamic expression of tumor PD-L1 is lower than that of exosome PD-L1 and the detection of tumor PD-L1 requires invasive tumor biopsies, exosome PD-L1 may be a promising blood-based biomarker. Subsequent confirmation of the clinical application potential of exosome PD-L1 in multiple cancer types is required. For example, Theodoraki et al. (2018) showed that in HNSCC patients, higher plasma levels of exosome PD-L1 were associated with stronger inhibition of CD8 effector $\mathrm{T}$ cell activation. Anti-PD-1 Abs significantly reduced the dose-dependent inhibition of $\mathrm{T}$ cell activity by exosome PD-L1 (Theodoraki et al., 2019). Poggio et al. (2019) found that exosome PD-L1 seems to be resistant to anti-PD-L1 treatment. At the same time, inhibiting exosome PD-L1 helps maintain long-lasting anti-tumor immunity (Zhang et al., 2017). A study by Del Re et al. (2018) explored the relationship between exosome PD-L1 mRNA expression and response to anti-PD- 1 treatment in melanoma $(n=18)$ and nonsmall cell lung cancer $(n=8)$. They emphasized that exosome PD-L1 should be considered when predicting the outcome of anti-PD-1 treatment (Siegel et al., 2012). This may also be the reason why the PD-L1 IHC profile of the tumor is not an ideal biomarker for the selection of anti-PD-1/PD-L1 immunotherapy candidates. Based on the above conclusions, we know that we cannot simply detect the tumor PD-L1 for verification. But if there is a serological marker that can provide reliable information on the expression status of tumor PD-L1, this situation can be greatly improved. The exosome PD-L1 is very likely to be this serological marker.

\section{REFERENCES}

Aubuchon, M., Bolt, L., Janssen-Heijnen, M., Verleisdonk-Bolhaar, S., van Marion, A., and van Berlo, C. (2017). Epidemiology, management and survival outcomes of primary cutaneous melanoma: a ten-year overview. Acta Chir. Belg. 117, 29-35.

Bergmann, C., Strauss, L., Zeidler, R., Lang, S., and Whiteside, T. L. (2007). Expansion of human $\mathrm{T}$ regulatory type 1 cells in the microenvironment of cyclooxygenase 2 overexpressing head and neck squamous cell carcinoma. Cancer Res. 67, 8865-8873. doi: 10.1158/0008-5472.Can-07-0767

\section{CONCLUSION}

Tumor-derived exosomes PD-L1 play a key role in tumor immune escape of tumors. At present, there are several mechanisms by which cell surface or exosome PD-L1 mediates tumor immunity to achieve immune escape, such as by inducing activated $\mathrm{T}$ cell apoptosis, promoting $\mathrm{T}$ cell weakness, enhancing the function of Tregs, inhibiting $\mathrm{T}$ cell proliferation, and inhibiting impaired T cell activation and IL-2 production.

Many studies have confirmed that PD-L1 on tumor cells mediates immunosuppressive effects. Similarly, PD-L1 secreted by tumor cells on the surface of exosomes binds to PD1 on $\mathrm{T}$ cells and exerts biological effects. However, further exploration of the molecular mechanisms is needed. Exosome PD-L1 has been shown to be of clinical value in the diagnosis, treatment and prognosis of various cancers, such as NSCLC, melanoma, gastric cancer, breast cancer and HNSCC. The measurement of PD-L1 levels in exosomes complements existing immune checkpoint measurements, facilitating the accuracy of immune-related tumor diagnosis. In addition, exosome PDL1 contributes to anti-PD-L1/PD-1 therapy and enhances the sensitivity of tumor patients to treatment. Nevertheless, more work remains to be done to apply tumor-derived exosome PD-L1 in clinical practice.

\section{AUTHOR CONTRIBUTIONS}

ZS provided direction and guidance throughout the preparation of this manuscript. BS and QD wrote and edited the manuscript. ZC, CC, QZ, and SH reviewed and made significant revisions of the manuscript. BQ, JL, GW, and WY collected and prepared the related manuscript. All authors read and approved the final manuscript.

\section{FUNDING}

This study was supported by the National Natural Science Foundation of China (81972663), Key Scientific Research Projects of Institutions of Higher Education in Henan Province (19A310024), Medical Scientific and Technological Research Project of Henan Province (201702027), National Natural Science Foundation of Henan Province (182300410342), and Health Commission Technology Talents Overseas Training Project of Henan Province (2018140).

Blank, C., Brown, I., Peterson, A. C., Spiotto, M., Iwai, Y., Honjo, T., et al. (2004). $\mathrm{PD}-\mathrm{L} 1 / \mathrm{B} 7 \mathrm{H}-1$ inhibits the effector phase of tumor rejection by $\mathrm{T}$ cell receptor (TCR) transgenic CD8+ T cells. Cancer Res. 64, 1140-1145.

Boukouris, S., and Mathivanan, S. (2015). Exosomes in bodily fluids are a highly stable resource of disease biomarkers. Proteomics Clin. Appl. 9, 358-367.

Brahmer, J. R., Rodríguez-Abreu, D., Robinson, A. G., Hui, R., Csőszi, T., Fülöp, A., et al. (2017). Health-related quality-of-life results for pembrolizumab versus chemotherapy in advanced, PD-L1-positive NSCLC (KEYNOTE-024): a multicentre, international, randomised, open-label phase 3 trial. Lancet Oncol. 18, 1600-1609. doi: 10.1016/s1470-2045(17)30690-3 
Bruttel, V. S., and Wischhusen, J. (2014). Cancer stem cell immunology: key to understanding tumorigenesis and tumor immune escape? Front. Immunol. 5:360. doi: 10.3389/fimmu.2014.00360

Cader, F. Z., Hu, X., Goh, W. L., Wienand, K., Ouyang, J., Mandato, E., et al. (2020). A peripheral immune signature of responsiveness to PD-1 blockade in patients with classical Hodgkin lymphoma. Nat. Med. 26, 1468-1479. doi: 10.1038/s41591-020-1006-1

Chatterjee, S., Chatterjee, A., Jana, S., Dey, S., Roy, H., Das, M. K., et al. (2020). Transforming growth factor beta orchestrates PD-L1 enrichment in tumor derived exosomes and mediates CD8 T cell dysfunction regulating early phosphorylation of TCR signalome in breast cancer. Carcinogenesis 42, 38-47. doi: 10.1093/carcin/bgaa092

Chen, G., Huang, A. C., Zhang, W., Zhang, G., Wu, M., Xu, W., et al. (2018). Exosome PD-L1 contributes to immunosuppression and is associated with anti-PD-1 response. Nature 560, 382-386. doi: 10.1038/s41586-018-0392-8

Chen, L., and Han, X. (2015). Anti-PD-1/PD-L1 therapy of human cancer: past, present, and future. J. Clin. Invest. 125, 3384-3391.

Chen, X. L., Yuan, S. X., Chen, C., Mao, Y. X., Xu, G., and Wang, X. Y. (2009). [Expression of B7-H1 protein in human pancreatic carcinoma tissues and its clinical significance]. Ai Zheng 28, 1328-1332. doi: 10.5732/cjc.009.10245

Colletti, M., Tomao, L., Galardi, A., Paolini, A., Di Paolo, V., De Stefanis, C., et al. (2020). Neuroblastoma-secreted exosomes carrying miR-375 promote osteogenic differentiation of bone-marrow mesenchymal stromal cells. J. Extracell. Vesicles 9:1774144. doi: 10.1080/20013078.2020.1774144

Colombo, M., Moita, C., van Niel, G., Kowal, J., Vigneron, J., Benaroch, P., et al. (2013). Analysis of ESCRT functions in exosome biogenesis, composition and secretion highlights the heterogeneity of extracellular vesicles. J. Cell Sci. 126(Pt 24), 5553-5565. doi: 10.1242/jcs. 128868

Colombo, M., Raposo, G., and Théry, C. (2014). Biogenesis, secretion, and intercellular interactions of exosomes and other extracellular vesicles. Annu. Rev. Cell Dev. Biol. 30, 255-289. doi: 10.1146/annurev-cellbio-101512-122326

Cordonnier, M., Chanteloup, G., Isambert, N., Seigneuric, R., Fumoleau, P., Garrido, C., et al. (2017). Exosomes in cancer theranostic: diamonds in the rough. Cell Adh. Migr. 11, 151-163. doi: 10.1080/19336918.2016.1250999

Cordonnier, M., Nardin, C., Chanteloup, G., Derangere, V., Algros, M. P., Arnould, L., et al. (2020). Tracking the evolution of circulating exosome-PD-L1 to monitor melanoma patients. J. Extracell. Vesicles 9:1710899. doi: 10.1080/ 20013078.2019.1710899

Daassi, D., Mahoney, K. M., and Freeman, G. J. (2020). The importance of exosome PDL1 in tumour immune evasion. Nat. Rev. Immunol. 20, 209-215. doi: 10. 1038/s41577-019-0264-y

Davies, L. C., Jenkins, S. J., Allen, J. E., and Taylor, P. R. (2013). Tissue-resident macrophages. Nat. Immunol. 14, 986-995. doi: 10.1038/ni.2705

Del Re, M., Marconcini, R., Pasquini, G., Rofi, E., Vivaldi, C., Bloise, F., et al. (2018). PD-L1 mRNA expression in plasma-derived exosomes is associated with response to anti-PD-1 antibodies in melanoma and NSCLC. Br. J. Cancer 118, 820-824. doi: 10.1038/bjc.2018.9

Dong, H., Strome, S., Salomao, D., Tamura, H., Hirano, F., Flies, D., et al. (2002). Tumor-associated $\mathrm{B} 7-\mathrm{H} 1$ promotes T-cell apoptosis: a potential mechanism of immune evasion. Nat. Med. 8, 793-800. doi: 10.1038/nm730

Dong, H., Zhu, G., Tamada, K., and Chen, L. (1999). B7-H1, a third member of the B7 family, co-stimulates T-cell proliferation and interleukin-10 secretion. Nat. Med. 5, 1365-1369. doi: 10.1038/70932

Eggermont, A. M. M., Blank, C. U., Mandala, M., Long, G. V., Atkinson, V., Dalle, S., et al. (2018). Adjuvant pembrolizumab versus placebo in resected stage III melanoma. N. Engl. J. Med. 378, 1789-1801. doi: 10.1056/NEJMoa1802357

Fan, Y., Che, X., Qu, J., Hou, K., Wen, T., Li, Z., et al. (2019). Exosome PD$\mathrm{L} 1$ retains immunosuppressive activity and is associated with gastric cancer prognosis. Ann. Surg. Oncol. 26, 3745-3755. doi: 10.1245/s10434-019-07431-7

Fehrenbacher, L., Spira, A., Ballinger, M., Kowanetz, M., Vansteenkiste, J., Mazieres, J., et al. (2016). Atezolizumab versus docetaxel for patients with previously treated non-small-cell lung cancer (POPLAR): a multicentre, open-label, phase 2 randomised controlled trial. Lancet 387, 1837-1846. doi: 10.1016/s01406736(16)00587-0

Ferlay, J., Steliarova-Foucher, E., Lortet-Tieulent, J., Rosso, S., Coebergh, J.-W. W., Comber, H., et al. (2013). Cancer incidence and mortality patterns in Europe: estimates for 40 countries in 2012. Eur. J. Cancer 49, 1374-1403.
Ferris, R. L., Whiteside, T. L., and Ferrone, S. (2006). Immune escape associated with functional defects in antigen-processing machinery in head and neck cancer. Clin. Cancer Res. 12, 3890-3895. doi: 10.1158/1078-0432.Ccr-05-2750

Foley, K., Kim, V., Jaffee, E., and Zheng, L. (2016). Current progress in immunotherapy for pancreatic cancer. Cancer Lett. 381, 244-251.

Frigola, X., Inman, B. A., Lohse, C. M., Krco, C. J., Cheville, J. C., Thompson, R. H., et al. (2011). Identification of a soluble form of B7-H1 that retains immunosuppressive activity and is associated with aggressive renal cell carcinoma. Clin. Cancer Res. 17, 1915-1923. doi: 10.1158/1078-0432.Ccr-100250

Funes, S. C., Rios, M., Escobar-Vera, J., and Kalergis, A. M. (2018). Implications of macrophage polarization in autoimmunity. Immunology 154, 186-195. doi: 10.1111/imm.12910

Gabrusiewicz, K., Li, X., Wei, J., Hashimoto, Y., Marisetty, A. L., Ott, M., et al. (2018). Glioblastoma stem cell-derived exosomes induce M2 macrophages and PD-L1 expression on human monocytes. Oncoimmunology 7:e1412909. doi: 10.1080/2162402x.2017.1412909

Geng, L., Huang, D., Liu, J., Qian, Y., Deng, J., Li, D., et al. (2008). B7-H1 upregulated expression in human pancreatic carcinoma tissue associates with tumor progression. J. Cancer Res. Clin. Oncol. 134, 1021-1027. doi: 10.1007/ s00432-008-0364-8

Grigg, C., and Rizvi, N. A. (2016). PD-L1 biomarker testing for non-small cell lung cancer: truth or fiction? J. Immunother. Cancer 4:48.

Guo, Y., Ji, X., Liu, J., Fan, D., Zhou, Q., Chen, C., et al. (2019). Effects of exosomes on pre-metastatic niche formation in tumors. Mol. Cancer 18:39. doi: 10.1186/s12943-019-0995-1

Haderk, F., Schulz, R., Iskar, M., Cid, L. L., Worst, T., Willmund, K. V., et al. (2017). Tumor-derived exosomes modulate PD-L1 expression in monocytes. Sci. Immunol. 2:eaah5509. doi: 10.1126/sciimmunol.aah5509

He, J., Hu, Y., Hu, M., and Li, B. (2015). Development of PD-1/PD-L1 pathway in tumor immune microenvironment and treatment for non-small cell lung cancer. Sci. Rep. 5, 1-9.

Herbst, R. S., Baas, P., Kim, D. W., Felip, E., Pérez-Gracia, J. L., Han, J. Y., et al. (2016). Pembrolizumab versus docetaxel for previously treated, PD-L1positive, advanced non-small-cell lung cancer (KEYNOTE-010): a randomised controlled trial. Lancet 387, 1540-1550. doi: 10.1016/s0140-6736(15)01281-7

Hessvik, N., and Llorente, A. (2018). Current knowledge on exosome biogenesis and release. Cell. Mol. Life Sci. 75, 193-208. doi: 10.1007/s00018-017-2595-9

Hong, C. S., Funk, S., Muller, L., Boyiadzis, M., and Whiteside, T. L. (2016). Isolation of biologically active and morphologically intact exosomes from plasma of patients with cancer. J. Extracell. Vesicles 5:29289. doi: 10.3402/jev. v5.29289

Hu, S., Li, Z., Lutz, H., Huang, K., Su, T., Cores, J., et al. (2020). Dermal exosomes containing miR-218-5p promote hair regeneration by regulating $\beta$-catenin signaling. Sci. Adv. 6:eaba1685. doi: 10.1126/sciadv.aba1685

Huang, H. H., Hsiao, F. Y., Chen, H. M., Wang, C. Y., and Ko, B. S. (2021). Antiviral prophylaxis for hepatitis B carriers improves the prognosis of diffuse large B-cell lymphoma in Taiwan-a population-based study. Br. J. Haematol. 192, 110-118. doi: 10.1111/bjh.17142

Huang, M., Yang, J., Wang, T., Song, J., Xia, J., Wu, L., et al. (2020). Homogeneous, low-volume, efficient and sensitive quantitation of circulating exosome PDL1 for cancer diagnosis and immunotherapy response prediction. Angewandte Chemie 59, 4800-4805.

Ichikawa, M., and Chen, L. (2005). Role of B7-H1 and B7-H4 molecules in down-regulating effector phase of T-cell immunity: novel cancer escaping mechanisms. Front. Biosci. 10:2856-2860. doi: 10.2741/1742

Iwai, Y., Ishida, M., Tanaka, Y., Okazaki, T., Honjo, T., and Minato, N. (2002). Involvement of PD-L1 on tumor cells in the escape from host immune system and tumor immunotherapy by PD-L1 blockade. Proc. Natl. Acad. Sci. U.S.A. 99, 12293-12297. doi: 10.1073/pnas.192461099

Keir, M., Butte, M., Freeman, G., and Sharpe, A. (2008). PD-1 and its ligands in tolerance and immunity. Annu. Rev. Immunol. 26, 677-704. doi: 10.1146/ annurev.immunol.26.021607.090331

Kim, D. H., Kim, H., Choi, Y. J., Kim, S. Y., Lee, J.-E., Sung, K. J., et al. (2019). Exosome PD-L1 promotes tumor growth through immune escape in non-small cell lung cancer. Exp. Mol. Med. 51, 1-13.

Koch, R., Demant, M., Aung, T., Diering, N., Cicholas, A., Chapuy, B., et al. (2014). Populational equilibrium through exosome-mediated Wnt signaling in 
tumor progression of diffuse large B-cell lymphoma. Blood 123, 2189-2198. doi: 10.1182/blood-2013-08-523886

Kourembanas, S. (2015). Exosomes: vehicles of intercellular signaling, biomarkers, and vectors of cell therapy. Annu. Rev. Physiol. 77, 13-27. doi: 10.1146/annurevphysiol-021014-071641

Li, C., Li, C., Zhi, C., Liang, W., Wang, X., Chen, X., et al. (2019). Clinical significance of PD-L1 expression in serum-derived exosomes in NSCLC patients. J. Transl. Med. 17:355. doi: 10.1186/s12967-019-2101-2

Li, J-W., Wei, P., Guo, Y., Shi, D., Yu, B-H., Su, Y-F., et al. (2020). Clinical significance of circulating exosomal PD-L1 and soluble PD-L1 in extranodal NK/T-cell lymphoma, nasal-type. Am. J. Cancer Res. 10, 4498-4512.

Li, Y., Zheng, Q., Bao, C., Li, S., Guo, W., Zhao, J., et al. (2015). Circular RNA is enriched and stable in exosomes: a promising biomarker for cancer diagnosis. Cell Res. 25, 981-984. doi: 10.1038/cr.2015.82

Lin, Y., Sung, W., Hsieh, M., Tsai, S., Lai, H., Yang, S., et al. (2015). High PD-L1 expression correlates with metastasis and poor prognosis in oral squamous cell carcinoma. PLoS One 10:e0142656. doi: 10.1371/journal.pone.0142656

Liu, C., Zeng, X., An, Z., Yang, Y., Eisenbaum, M., Gu, X., et al. (2018). Sensitive detection of exosome proteins via a compact surface plasmon resonance biosensor for cancer diagnosis. ACS Sens. 3, 1471-1479. doi: 10.1021/acssensors. 8 b00230

Liu, N., Zhang, J., Yin, M., Liu, H., Zhang, X., Li, J., et al. (2021). Inhibition of xCT suppresses the efficacy of anti-PD-1/L1 melanoma treatment through exosome PD-L1-induced macrophage M2 polarization. Mol. Ther. 29, 2321-2334. doi: 10.1016/j.ymthe.2021.03.013

Lo Cicero, A., Stahl, P., and Raposo, G. (2015). Extracellular vesicles shuffling intercellular messages: for good or for bad. Curr. Opin. Cell Biol. 35, 69-77. doi: 10.1016/j.ceb.2015.04.013

Ludwig, S., Floros, T., Theodoraki, M. N., Hong, C. S., Jackson, E. K., Lang, S., et al. (2017). Suppression of lymphocyte functions by plasma exosomes correlates with disease activity in patients with head and neck cancer. Clin. Cancer Res. 23, 4843-4854. doi: 10.1158/1078-0432.Ccr-16-2819

Lux, A., Kahlert, C., Grützmann, R., and Pilarsky, C. (2019). C-met and PD-L1 on circulating exosomes as diagnostic and prognostic markers for pancreatic Cancer. Int. J. Mol. Sci. 20:3305.

Martinez, F. O., and Gordon, S. (2014). The M1 and M2 paradigm of macrophage activation: time for reassessment. F1000Prime Rep. 6:13. doi: 10.12703/p6-13

Martinez, V. G., O’Neill, S., Salimu, J., Breslin, S., Clayton, A., Crown, J., et al. (2017). Resistance to HER2-targeted anti-cancer drugs is associated with immune evasion in cancer cells and their derived extracellular vesicles. Oncoimmunology 6:e1362530.

Mashouri, L., Yousefi, H., Aref, A., Ahadi, A., Molaei, F., and Alahari, S. (2019). Exosomes: composition, biogenesis, and mechanisms in cancer metastasis and drug resistance. Mol. Cancer 18:75. doi: 10.1186/s12943-019-0991-5

Melo, S. A., Luecke, L. B., Kahlert, C., Fernandez, A. F., Gammon, S. T., Kaye, J., et al. (2015). Glypican-1 identifies cancer exosomes and detects early pancreatic cancer. Nature 523, 177-182. doi: 10.1038/nature14581

Mittal, D., Gubin, M. M., Schreiber, R. D., and Smyth, M. J. (2014). New insights into cancer immunoediting and its three component phases-elimination, equilibrium and escape. Curr. Opin. Immunol. 27, 16-25. doi: 10.1016/j.coi. 2014.01.004

Mittendorf, E. A., Philips, A. V., Meric-Bernstam, F., Qiao, N., Wu, Y., Harrington, S., et al. (2014). PD-L1 expression in triple-negative breast cancer. Cancer Immunol. Res. 2, 361-370. doi: 10.1158/2326-6066.Cir-13-0127

Mok, T. S. K., Wu, Y. L., Kudaba, I., Kowalski, D. M., Cho, B. C., Turna, H. Z., et al. (2019). Pembrolizumab versus chemotherapy for previously untreated, PD-L1-expressing, locally advanced or metastatic non-small-cell lung cancer (KEYNOTE-042): a randomised, open-label, controlled, phase 3 trial. Lancet 393, 1819-1830. doi: 10.1016/s0140-6736(18)32409-7

Monypenny, J., Milewicz, H., Flores-Borja, F., Weitsman, G., Cheung, A., Chowdhury, R., et al. (2018). ALIX Regulates tumor-mediated immunosuppression by controlling EGFR activity and PD-L1 presentation. Cell Rep. 24, 630-641. doi: 10.1016/j.celrep.2018.06.066

Mourad, M., Jetmore, T., Jategaonkar, A. A., Moubayed, S., Moshier, E., and Urken, M. L. (2017). Epidemiological trends of head and neck cancer in the United States: a SEER population study. J. Oral Maxillofac. Surg. 75, 2562-2572.

Muro, K., Chung, H. C., Shankaran, V., Geva, R., Catenacci, D., Gupta, S., et al. (2016). Pembrolizumab for patients with PD-L1-positive advanced gastric cancer (KEYNOTE-012): a multicentre, open-label, phase 1b trial. Lancet Oncol. $17,717-726$.

Nagato, T., Ohkuri, T., Ohara, K., Hirata, Y., Kishibe, K., Komabayashi, Y., et al. (2017). Programmed death-ligand 1 and its soluble form are highly expressed in nasal natural killer/T-cell lymphoma: a potential rationale for immunotherapy. Cancer Immunol. Immunother. 66, 877-890. doi: 10.1007/s00262-0171987-x

Nahta, R., and Esteva, F. J. (2007). Trastuzumab: triumphs and tribulations. Oncogene 26, 3637-3643.

Nawaz, M., Camussi, G., Valadi, H., Nazarenko, I., Ekström, K., Wang, X., et al. (2014). The emerging role of extracellular vesicles as biomarkers for urogenital cancers. Nat. Rev. Urol. 11, 688-701. doi: 10.1038/nrurol.2014. 301

Nishimura, H., Agata, Y., Kawasaki, A., Sato, M., Imamura, S., Minato, N., et al. (1996). Developmentally regulated expression of the PD-1 protein on the surface of double-negative (CD4-CD8-) thymocytes. Int. Immunol. 8, 773-780. doi: 10.1093/intimm/8.5.773

Noguchi, T., Ward, J. P., Gubin, M. M., Arthur, C. D., Lee, S. H., Hundal, J., et al. (2017). Temporally distinct PD-L1 expression by tumor and host cells contributes to immune escape. Cancer Immunol. Res. 5, 106-117. doi: 10.1158/ 2326-6066.Cir-16-0391

Nolte-'t Hoen, E., Buermans, H., Waasdorp, M., Stoorvogel, W., Wauben, M., and Hoen, P. A. (2012). Deep sequencing of RNA from immune cell-derived vesicles uncovers the selective incorporation of small non-coding RNA biotypes with potential regulatory functions. Nucleic Acids Res. 40, 9272-9285. doi: 10.1093/ nar/gks658

Okazaki, F., Matsunaga, N., Hamamura, K., Suzuki, K., Nakao, T., Okazaki, H., et al. (2017). Administering xCT inhibitors based on circadian clock improves antitumor effects. Cancer Res. 77, 6603-6613. doi: 10.1158/0008-5472.Can-170720

Ono, K., Sogawa, C., Kawai, H., Tran, M., Taha, E., Lu, Y., et al. (2020). Triple knockdown of CDC37, HSP90-alpha and HSP90-beta diminishes extracellular vesicles-driven malignancy events and macrophage M2 polarization in oral cancer. J. Extracell. Vesicles 9:1769373. doi: 10.1080/20013078.2020.176 9373

Pang, Y., Shi, J., Yang, X., Wang, C., Sun, Z., and Xiao, R. (2020). Personalized detection of circling exosome PD-L1 based on Fe3O4@ TiO2 isolation and SERS immunoassay. Biosens. Bioelectron. 148:111800.

Patel, S. P., and Kurzrock, R. (2015). PD-L1 expression as a predictive biomarker in cancer immunotherapy. Mol. Cancer Ther. 14, 847-856. doi: 10.1158/15357163.Mct-14-0983

Poggio, M., Hu, T., Pai, C. C., Chu, B., Belair, C. D., Chang, A., et al. (2019). Suppression of exosome PD-L1 induces systemic anti-tumor immunity and memory. Cell 177, 414-427.e13. doi: 10.1016/j.cell.2019.02.016

Price, T. J., Shapiro, J. D., Segelov, E., Karapetis, C. S., Pavlakis, N., Van Cutsem, E., et al. (2012). Management of advanced gastric cancer. Expert Rev. Gastroenterol. Hepatol. 6, 199-209.

Rahbari, M., Rahbari, N., Reissfelder, C., Weitz, J., and Kahlert, C. (2016). Exosomes: novel implications in diagnosis and treatment of gastrointestinal cancer. Langenbecks Arch. Surg. 401, 1097-1110.

Ramirez, M. I., Amorim, M. G., Gadelha, C., Milic, I., Welsh, J. A., Freitas, V. M., et al. (2018). Technical challenges of working with extracellular vesicles. Nanoscale 10, 881-906.

Ribas, A., Hamid, O., Daud, A., Hodi, F. S., Wolchok, J. D., Kefford, R., et al. (2016). Association of pembrolizumab with tumor response and survival among patients with advanced melanoma. JAMA 315, 1600-1609. doi: 10.1001/jama. 2016.4059

Ricklefs, F. L., Alayo, Q., Krenzlin, H., Mahmoud, A. B., Speranza, M. C., Nakashima, H., et al. (2018). Immune evasion mediated by PD-L1 on glioblastoma-derived extracellular vesicles. Sci. Adv. 4:eaar2766. doi: 10.1126/ sciadv.aar2766

Roefs, M., Sluijter, J., and Vader, P. (2020). Extracellular vesicle-associated proteins in tissue repair. Trends Cell Biol. 30, 990-1013. doi: 10.1016/j.tcb.2020. 09.009

Shi, M. H., Xing, Y. F., Zhang, Z. L., Huang, J. A., and Chen, Y. J. (2013). [Effect of soluble PD-L1 released by lung cancer cells in regulating the function of T lymphocytes]. Zhonghua Zhong Liu Za Zhi 35, 85-88. doi: 10.3760/cma.j.issn. 0253-3766.2013.02.002 
Siegel, R., Naishadham, D., and Jemal, A. (2012). Cancer statistics, 2012. CA Cancer J. Clin. 62, 10-29.

Slamon, D. J., Godolphin, W., Jones, L. A., Holt, J. A., Wong, S. G., Keith, D. E., et al. (1989). Studies of the HER-2/neu proto-oncogene in human breast and ovarian cancer. Science 244, 707-712.

Steinman, R. M., and Banchereau, J. (2007). Taking dendritic cells into medicine. Nature 449, 419-426.

Strome, S. E., Dong, H., Tamura, H., Voss, S. G., Flies, D. B., Tamada, K., et al. (2003). B7-H1 blockade augments adoptive T-cell immunotherapy for squamous cell carcinoma. Cancer Res. 63, 6501-6505.

Stuffers, S., Sem Wegner, C., Stenmark, H., and Brech, A. (2009). Multivesicular endosome biogenesis in the absence of ESCRTs. Traffic 10, 925-937. doi: 10. $1111 / j .1600-0854.2009 .00920 . x$

Sun, C., Mezzadra, R., and Schumacher, T. N. (2018). Regulation and function of the PD-L1 checkpoint. Immunity 48, 434-452. doi: 10.1016/j.immuni.2018. 03.014

Tang, M. K., and Wong, A. S. (2015). Exosomes: emerging biomarkers and targets for ovarian cancer. Cancer Lett. 367, 26-33. doi: 10.1016/j.canlet.2015.07.014

Taube, J. M., Klein, A., Brahmer, J. R., Xu, H., Pan, X., Kim, J. H., et al. (2014). Association of PD-1, PD-1 ligands, and other features of the tumor immune microenvironment with response to anti-PD-1 therapy. Clin. Cancer Res. 20, 5064-5074. doi: 10.1158/1078-0432.Ccr-13-3271

Thakur, A., Qiu, G., Xu, C., Han, X., Yang, T., Ng, S. P., et al. (2020). Label-free sensing of exosome MCT1 and CD147 for tracking metabolic reprogramming and malignant progression in glioma. Sci. Adv. 6:eaaz6119. doi: 10.1126/sciadv. aaz6119

Thakur, B., Zhang, H., Becker, A., Matei, I., Huang, Y., Costa-Silva, B., et al. (2014). Double-stranded DNA in exosomes: a novel biomarker in cancer detection. Cell Res. 24, 766-769. doi: 10.1038/cr.2014.44

Theodoraki, M. N., Yerneni, S. S., Hoffmann, T. K., Gooding, W. E., and Whiteside, T. L. (2018). Clinical significance of PD-L1(+) exosomes in plasma of head and neck cancer patients. Clin. Cancer Res. 24, 896-905. doi: 10.1158/1078-0432. Ccr-17-2664

Theodoraki, M. N., Yerneni, S., Gooding, W. E., Ohr, J., Clump, D. A., Bauman, J. E., et al. (2019). Circulating exosomes measure responses to therapy in head and neck cancer patients treated with cetuximab, ipilimumab, and IMRT. Oncoimmunology 8:1593805. doi: 10.1080/2162402x.2019.1593805

Théry, C., Zitvogel, L., and Amigorena, S. (2002). Exosomes: composition, biogenesis and function. Nat. Rev. Immunol. 2, 569-579. doi: 10.1038/nri855

Topalian, S. L., Taube, J. M., Anders, R. A., and Pardoll, D. M. (2016). Mechanismdriven biomarkers to guide immune checkpoint blockade in cancer therapy. Nat. Rev. Cancer 16:275.

van Niel, G., D’Angelo, G., and Raposo, G. (2018). Shedding light on the cell biology of extracellular vesicles. Nat. Rev. Mol. Cell Biol. 19, 213-228. doi: 10.1038/nrm.2017.125

Villarroya-Beltri, C., Gutiérrez-Vázquez, C., Sánchez-Cabo, F., Pérez-Hernández, D., Vázquez, J., Martin-Cofreces, N., et al. (2013). Sumoylated hnRNPA2B1 controls the sorting of miRNAs into exosomes through binding to specific motifs. Nat. Commun. 4:2980. doi: 10.1038/ncomms3980

Wang, L., Ma, Q., Chen, X., Guo, K., Li, J., and Zhang, M. (2010). Clinical significance of B7-H1 and B7-1 expressions in pancreatic carcinoma. World J. Surg. 34, 1059-1065. doi: 10.1007/s00268-010-0448-x

Wang, X., Teng, F., Kong, L., and Yu, J. (2016). PD-L1 expression in human cancers and its association with clinical outcomes. OncoTargets Ther. 9, 5023-5039. doi: $10.2147 /$ ott.S105862
Welton, J. L., Khanna, S., Giles, P. J., Brennan, P., Brewis, I. A., Staffurth, J., et al. (2010). Proteomics analysis of bladder cancer exosomes. Mol. Cell. Proteomics 9, 1324-1338. doi: 10.1074/mcp.M000063-MCP201

Wen, Z. F., Liu, H., Gao, R., Zhou, M., Ma, J., Zhang, Y., et al. (2018). Tumor cell-released autophagosomes (TRAPs) promote immunosuppression through induction of M2-like macrophages with increased expression of PD-L1. J. Immunother. Cancer 6:151. doi: 10.1186/s40425-018-0452-5

Whiteside, T. L. (2016). Exosomes and tumor-mediated immune suppression. J. Clin. Invest. 126, 1216-1223. doi: 10.1172/jci81136

Whiteside, T. L. (2018). Head and neck carcinoma immunotherapy: facts and hopes. Clin. Cancer Res. 24, 6-13. doi: 10.1158/1078-0432.Ccr-17-1261

Xiang, S. Y., Yu, C., Aggarwal, A., and Reinhard, C. (2016). Genomic alterations and molecular subtypes of gastric cancers in Asians. Chin. J. Cancer 35:42.

Xing, Y. F., Zhang, Z. L., Shi, M. H., Ma, Y., and Chen, Y. J. (2012). [The level of soluble programmed death-1 in peripheral blood of patients with lung cancer and its clinical implications]. Zhonghua Jie He He Hu Xi Za Zhi 35, 102-106.

Yang, Y., Li, C.-W., Chan, L.-C., Wei, Y., Hsu, J.-M., Xia, W., et al. (2018). Exosome PD-L1 harbors active defense function to suppress T cell killing of breast cancer cells and promote tumor growth. Cell Res. 28, 862-864.

Zahn, M., Kaluszniak, B., Möller, P., and Marienfeld, R. (2020). The PTP1B mutant $\mathrm{PTP} 1 \mathrm{~B} \triangle 2-4$ is a positive regulator of the JAK/STAT signalling pathway in Hodgkin lymphoma. Carcinogenesis 42, 517-527. doi: 10.1093/carcin/bgaa144

Zaretsky, J. M., Garcia-Diaz, A., Shin, D. S., Escuin-Ordinas, H., Hugo, W., HuLieskovan, S., et al. (2016). Mutations associated with acquired resistance to PD-1 blockade in melanoma. N. Engl. J. Med. 375, 819-829. doi: 10.1056/ NEJMoa1604958

Zhang, J., Gao, J., Li, Y., Nie, J., Dai, L., Hu, W., et al. (2015). Circulating PD-L1 in NSCLC patients and the correlation between the level of PD-L1 expression and the clinical characteristics. Thorac. Cancer 6, 534-538. doi: 10.1111/1759-7714. 12247

Zhang, Y., Du, W., Chen, Z., and Xiang, C. (2017). Upregulation of PD-L1 by SPP1 mediates macrophage polarization and facilitates immune escape in lung adenocarcinoma. Exp. Cell Res. 359, 449-457. doi: 10.1016/j.yexcr.2017.08.028

Zhou, Y., Miao, J., Wu, H., Tang, H., Kuang, J., Zhou, X., et al. (2017). PD-1 and PDL1 expression in 132 recurrent nasopharyngeal carcinoma: the correlation with anemia and outcomes. Oncotarget 8, 51210-51223. doi: 10.18632/oncotarget. 17214

Conflict of Interest: The authors declare that the research was conducted in the absence of any commercial or financial relationships that could be construed as a potential conflict of interest.

Publisher's Note: All claims expressed in this article are solely those of the authors and do not necessarily represent those of their affiliated organizations, or those of the publisher, the editors and the reviewers. Any product that may be evaluated in this article, or claim that may be made by its manufacturer, is not guaranteed or endorsed by the publisher.

Copyright ( 2021 Shao, Dang, Chen, Chen, Zhou, Qiao, Liu, Hu, Wang, Yuan and Sun. This is an open-access article distributed under the terms of the Creative Commons Attribution License (CC BY). The use, distribution or reproduction in other forums is permitted, provided the original author(s) and the copyright owner(s) are credited and that the original publication in this journal is cited, in accordance with accepted academic practice. No use, distribution or reproduction is permitted which does not comply with these terms. 